\title{
Limited subtotal gastrectomy for early remnant gastric cancer
}

\author{
Yuichi Hosokawa • Masaru Konishi - Yatsuka Sahara • \\ Takahiro Kinoshita $\cdot$ Shinichiro Takahashi $\cdot$ Naoto Gotohda \\ Yuichiro Kato • Taira Kinoshita
}

Received: 9 February 2013/ Accepted: 11 June 2013/Published online: 6 July 2013

(C) The International Gastric Cancer Association and The Japanese Gastric Cancer Association 2013

\begin{abstract}
Background Detection of early remnant gastric cancer (ERGC) is increasing as a result of the development of endoscopic technology and a surveillance program. The aim of this study was to evaluate the results of limited subtotal gastrectomy (SG) surgery for ERGC compared to total gastrectomy (TG).

Methods We retrospectively reviewed a database of 72 consecutive patients with remnant gastric cancer who underwent laparotomy at the National Cancer Center Hospital East between January 1993 and December 2008. Thirty-five patients with a preoperative diagnosis of ERGC underwent curative resection: 13 SG and 22 conventional TG. Patients and tumor characteristics, operative results, and postoperative assessments 1 year after surgery were compared between the two groups.

Results Operating time, blood transfusion, and hospital stay were similar in the two groups. In the SG group, blood loss and postoperative recovery of body weight tended to be better than in the TG group. There was no dumping syndrome in the SG group, while this occurred in three patients in the TG group. The levels of hemoglobin and total protein were higher 1 year after remnant gastrectomy in the SG group than in the TG group. No recurrence of gastric cancer was detected in the SG group during median follow-up of 99.2 months.
\end{abstract}

Y. Hosokawa $(\bowtie) \cdot$ M. Konishi · Y. Sahara · T. Kinoshita · S. Takahashi - N. Gotohda - Y. Kato - T. Kinoshita Department of Surgical Oncology, National Cancer Center Hospital East, 6-5-1 Kashiwanoha, Kashiwa 277-8577, Japan e-mail: ryu37072002@yahoo.co.jp
Conclusion In comparison to TG, limited SG surgery for ERGC improved the postoperative course, with no recurrence of cancer. Therefore, SG is a safe and effective treatment for ERGC.

Keywords Limited surgery - Early gastric cancer . Remnant gastric cancer

\section{Introduction}

Remnant gastric cancer (RGC) was first reported by Balfour [1] and has been recognized as an important cancer model. Many previous studies have demonstrated the clinical and histopathological features of RGC, and that distal gastrectomy appears to increase the risk of its development, especially at the anastomosis site and suture line [1-7]. Although there have been no guidelines for surgical treatment of RGC, it is usually detected at an advanced stage, and total gastrectomy (TG) has been accepted for a long time. At present, detection of early remnant gastric cancer (ERGC) is increasing as a result of the development of endoscopic technology and a surveillance program with periodic endoscopy [8-10]. Endoscopic mucosal resection and submucosal dissection are applicable for treatment of ERGC; however, endoscopic treatment in the remnant stomach is technically difficult because of the narrow inner space and presence of massive fibrosis around the suture line [11].

We have used subtotal gastrectomy (SG) as minimally invasive surgery for ERGC that did not meet endoscopic treatment criteria. In the present retrospective study, we compared outcomes of SG and TG for ERGC to clarify the availability of SG. This is believed to be the first report of limited surgery for RGC. 


\section{Patinets and methods}

We retrospectively reviewed a database of 72 consecutive patients with RGCs who underwent laparotomy at the National Cancer Center Hospital East between January 1993 and December 2008. Thirty-five patients with preoperative diagnosis of ERGC underwent curative resection: 13 with SG and 22 with conventional TG.

Indications for SG comprised mucosal or submucosal RGC without lymph node metastasis, for which endoscopic resection was not indicated by preoperative diagnosis. The operation time interval and initial disease did not affect the adoption of SG. From the anatomical perspective, we determined the oral resection line at a distance of $>2 \mathrm{~cm}$ from the tumor, and the distance from the oral resection line to the esophagogastric junction should be sufficiently maintained. In the criteria, the tumor would to be completely resected by subtotal gastrectomy. With the above indications, initial gastrectomy was inevitably antrectomy for gastric or duodenal ulcer as the left gastric artery remaining.

We evaluated patient and tumor characteristics, operative details, postoperative results, morbidity, mortality, and cancer recurrence. Nutritional and gastrointestinal status 1 year after remnant gastrectomy was evaluated by self reports of dumping syndrome, body weight change, and laboratory data. We compared these data between the SG and TG groups. The number of regional lymph node stations was categorized according to the Japanese classification of gastric carcinoma [12].

SG for ERGC was performed as follows (Figs. 1, 2). (1) Extent of resection: subtotal gastrectomy with the margin $>2 \mathrm{~cm}$ from the cancer, including the anastomosis of the first operation (gastroduodenostomy or gastrojejunostomy). (2) Lymph node dissection: along the anastomotic duodenum or jejunum, left gastric artery (station 7), left gastroepiploic vessels (station 4sb), lesser curvature (station 3), and right paracardial region (station 1). Lymph nodes along the common hepatic artery (station 8a) and proximal splenic artery (station 11p) were dissected in some cases. (3) Reconstruction: the Roux-en Y method was usually used. For a patient whose previous operation method was Billroth I, this was rarely used again.

TG for ERGC was performed according to the conventional procedure with preservation of the spleen. Gastrointestinal reconstruction was performed with the Rouxen Y method.

Statistical analysis was performed by the MannWhitney $U$ test, $t$ test, and $\chi^{2}$ test. All statistical analyses were performed using SPSS for Windows (SPSS Inc., Tokyo, Japan). $P<0.05$ was considered statistically significant.

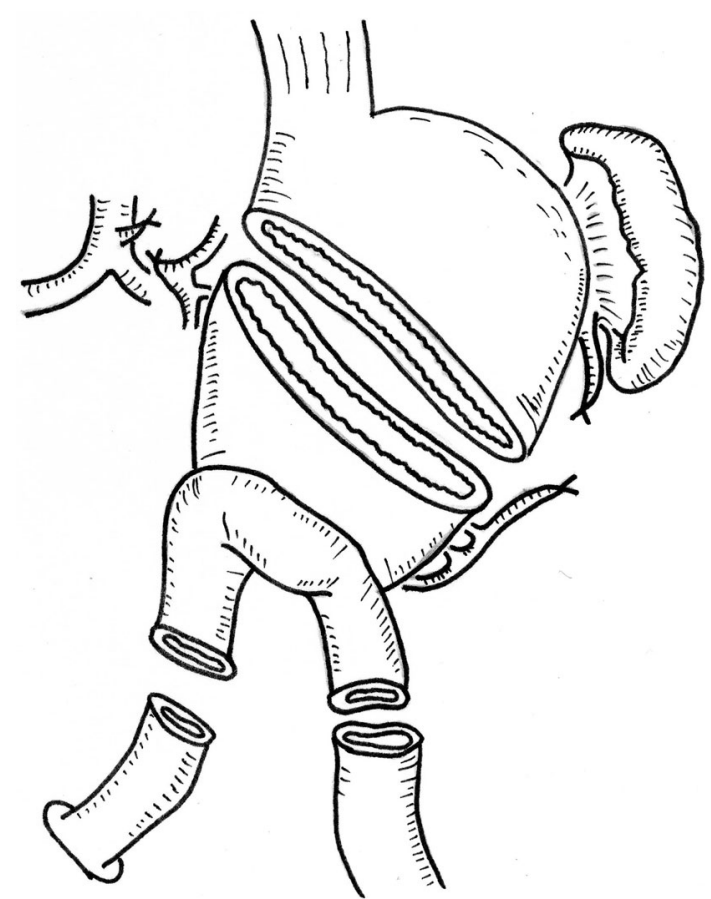

Fig. 1 Operative procedure of SG for RGC after BII operation

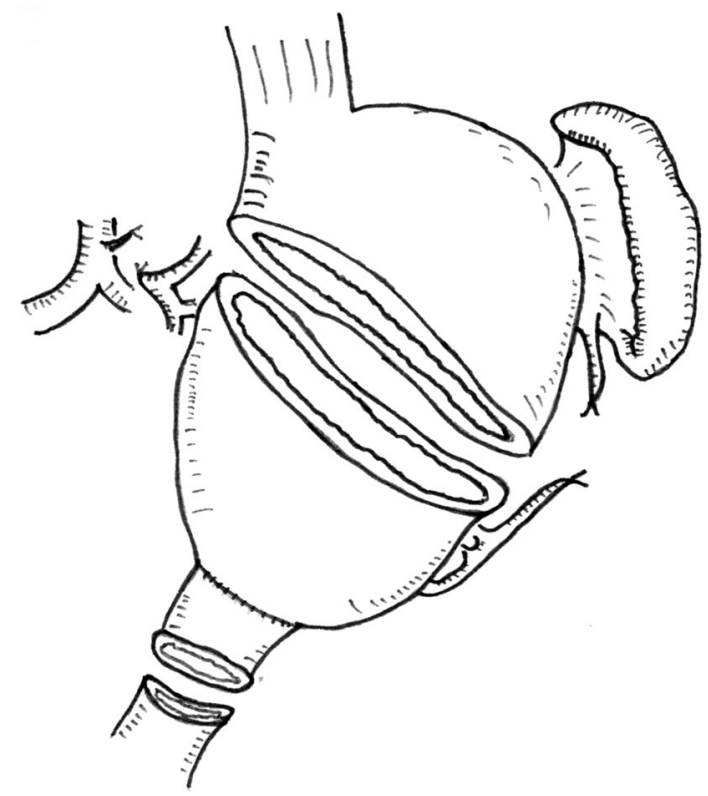

Fig. 2 Operative procedure of SG for RGC after BI operation

\section{Results}

Among 35 ERGC patients, 13 underwent SG and 22 conventional TG. The patient and tumor characteristics are shown in Table 1. There were no significant differences in age and sex between the two groups. In the SG group, initial disease was benign in all cases, and the operation time interval was longer. With regard to tumor location, the 
Table 1 Baseline characteristics of patients and tumors

\begin{tabular}{|c|c|c|c|}
\hline & $\mathrm{SG}(n=13)$ & TG $(n=22)$ & $P$ valu \\
\hline Age (years) & $66.1 \pm 6 \mathrm{D}$ & $67.6 \pm 103$ & 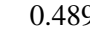 \\
\hline $\operatorname{Sex}(M / F)$ & $13 / 0$ & $17 / 5$ & 0.06 \\
\hline \multicolumn{4}{|l|}{ Initial disease } \\
\hline Benign & $13(100 \%)$ & $6(27.2 \%)$ & \multirow[t]{2}{*}{$<0.00$} \\
\hline Cancer & 0 & $16(72.8 \%)$ & \\
\hline \multicolumn{4}{|l|}{ Previous operation procedure } \\
\hline B-I & $4(30.7 \%)$ & $14(63.6 \%)$ & \multirow[t]{2}{*}{0.06} \\
\hline B-II & $9(69.3 \%)$ & $8(36.4 \%)$ & \\
\hline Time interval (year) & $37.0 \pm 87$ & $14.6 \pm 10.9$ & $<0.00$ \\
\hline \multicolumn{4}{|l|}{ Tumor location } \\
\hline Anastomotic site & $10(76.9 \%)$ & $6(27.3 \%)$ & \multirow[t]{3}{*}{0.01} \\
\hline Suture line & $2(15.4 \%)$ & $4(18.2 \%)$ & \\
\hline Non-stump area & $1(7.7 \%)$ & $12(54.5 \%)$ & \\
\hline \multicolumn{4}{|l|}{ Histological tumor depth } \\
\hline Mucosa & $7(53.8 \%)$ & $10(45.5 \%)$ & \multirow[t]{3}{*}{0.31} \\
\hline Submucosa & $3(23.1 \%)$ & $10(45.5 \%)$ & \\
\hline Muscularis propria & $3(23.1 \%)$ & $2(9.0 \%)$ & \\
\hline \multicolumn{4}{|l|}{ Lymph node metastasis } \\
\hline Negative & $13(100 \%)$ & $20(90.9 \%)$ & \multirow[t]{2}{*}{0.26} \\
\hline Positive & 0 & $2(9.1 \%)$ & \\
\hline \multicolumn{4}{|l|}{ Histopathological glade } \\
\hline Differentiated & $10(76.9 \%)$ & $12(54.5 \%)$ & \multirow[t]{2}{*}{0.186} \\
\hline Undifferentiated & $3(23.1 \%)$ & $10(45.5 \%)$ & \\
\hline \multicolumn{4}{|l|}{ Laboratory data } \\
\hline $\operatorname{Hemoglobin}(\mathrm{g} / \mathrm{dl})$ & $13.0 \pm 0.9$ & $12.3 \pm 1.3$ & 0.10 \\
\hline Lymphocytes $\left(/ \mathrm{mm}^{3}\right)$ & $1350 \pm 517$ & $1302 \pm 388$ & 0.78 \\
\hline Total protein $(\mathrm{g} / \mathrm{dl})$ & $6.9 \pm 0.6$ & $6.6 \pm 0.5$ & 0.09 \\
\hline Alubumin (g/dl) & $4.0 \pm 0.3$ & $3.9 \pm 0.4$ & 0.28 \\
\hline Total cholesterol (mg/dl) & $202 \pm 31.5$ & $187 \pm 32.5$ & 0.16 \\
\hline
\end{tabular}

anastomotic site was significantly larger in the SG group. With the preoperative diagnosis of the tumor depth as mucosal or submucosal, three patients in the SG group and two in the TG group showed muscularis propria invasion. In the TG group, there were two patients with lymph node metastasis. The lymph node stations were along the short gastric artery and left paracardial lymph node; however, both tumors were located in the cardia. There were no significant differences in preoperative laboratory data between the two groups.

Operative findings are shown in Table 2. Operation time, blood transfusion, and hospital stay were similar in the two groups. In the SG group, blood loss was lower than in the TG group, although the difference was not significant. Postoperative complications were similar between the two groups; however, there was no anastomotic leakage in the SG group. There was no mortality in either group.

Postoperative findings 1 year after surgery are shown in Table 3. In the SG group, postoperative recovery of body
Table 2 Operative results for each group

\begin{tabular}{llll}
\hline & SG $(n=13)$ & TG $(n=22)$ & $P$ value \\
\hline Operating time (min) & $174 \pm 53.0$ & $200 \pm 59.8$ & 0.229 \\
Blood loss (ml) & $381 \pm 372$ & $597 \pm 489$ & 0.079 \\
Blood transfusion & & & \\
$\quad$ No & $12(92.3 \%)$ & $19(86.4 \%)$ & 0.593 \\
Yes & $1(7.7 \%)$ & $3(13.6 \%)$ & \\
Hospital stay (days) & $17.8 \pm 8.7$ & $18.6 \pm 7.1$ & 0.468 \\
Complication & $3(23.1 \%)$ & $6(27.3 \%)$ & 0.832 \\
Wound infection & 0 & 2 & \\
Anastomotic stenosis & 1 & 1 & \\
Anastomotic leakage & 0 & 1 & \\
Hemorrhage & 1 & 0 & \\
Pancreatic fistula & 1 & 2 & \\
Mortality & 0 & 0 & \\
\hline
\end{tabular}

Table 3 Postoperative assessments 1 year after surgery and recurrence

\begin{tabular}{llll}
\hline & $\begin{array}{l}\text { SG } \\
(n=13)\end{array}$ & $\begin{array}{l}\text { TG } \\
(n=22)\end{array}$ & $P$ value \\
\hline $\begin{array}{l}\text { Dumping syndrome } \\
\text { Body weight change ratio } \\
(\%)\end{array}$ & 0 & $3(13.6 \%)$ & 0.279 \\
Laboratory data & & $94.1 \pm 7.7$ & 0.155 \\
$\quad$ Hemoglobin $(\mathrm{g} / \mathrm{dl})$ & $12.9 \pm 1.0$ & $11.7 \pm 1.5$ & 0.013 \\
Lymphocytes $\left(/ \mathrm{mm}^{3}\right)$ & $1236 \pm 219$ & $1346 \pm 461$ & 0.623 \\
Total protein $(\mathrm{g} / \mathrm{dl})$ & $6.9+0.4$ & $6.6 \pm 0.3$ & 0.016 \\
Alubumin $(\mathrm{g} / \mathrm{dl})$ & $4.0 \pm 0.3$ & $3.9 \pm 0.4$ & 0.287 \\
Total cholesterol $(\mathrm{mg} / \mathrm{dl})$ & $192 \pm 36.6$ & $176 \pm 33.9$ & 0.345 \\
Recurrence & 0 & $1(4.5 \%)$ & 0.446 \\
\hline
\end{tabular}

weight was better than in the TG group, with no significant difference. There was no dumping syndrome in the SG group. For laboratory data, hemoglobin and total protein were significantly higher in the SG group. There was no recurrence of cancer during median follow-up of 99.2 months (range 25-169 months) in the SG group, and one recurrence of liver metastasis during median follow-up of 57.2 months (range 11-169 months) in the TG group.

\section{Discussion}

We investigated the surgical techniques and outcomes, and postoperative quality of life in patients who underwent SG or TG for ERGC. We showed several advantages of SG over conventional TG for ERGC.

For many years, TG has been used for treatment of RGC because the tumors are usually detected at an advanced stage. However, limited surgery should be selected for 
ERGC for which endoscopic treatment is not indicated. Previous studies have presented the clinical and histopathological features of RGC, and SG was partly carried out in some cases [13-16]. Although limited surgery such as segmental gastrectomy for primary early gastric cancer has often been reported [17-21], there have been no reports about limited surgery for ERGC.

In our institution, endoscopic treatment for $\mathrm{RGC}$ is indicated for intramucosal cancer with no lymph node metastasis, which is confirmed as well or moderately differentiated adenocarcinoma. Tumors without the above indications are usually treated surgically. In some surgical methods, SG should be selected for a patient whose tumor depth is mucosal or submucosal without lymph node metastasis. SG should also be selected for a patient whose tumor can be resected with a sufficient margin. For RGC that surrounds an anastomotic site, the preoperative diagnosis for invasion depth and endoscopic treatment is difficult because of massive fibrosis around the suture line [11]. A remnant gastric cancer with the preoperative diagnosis of muscularis propia in depth might be deeper in the pathological diagnosis of the resected specimen. We consider that SG for RGC is absolutely limited surgery for early stage cancer, and advanced remnant gastric cancer should be treated by total gastrectomy with a sufficient margin and sufficient lymph node dissection. Therefore, ERGC that surrounds an anastomosis is often an indication for SG. The size of the remnant stomach in disease that was initially benign tends to be larger, and a patient whose initial disease is benign and whose tumor surrounds an anastomosis is most suitable for SG. With our indications for $\mathrm{SG}$, the left gastric artery remained in all patients in the SG group. In fact, we adopted SG for ERGC with the concept of distal gastrectomy instead of total gastrectomy after anterectomy. Lymph node dissection is another important consideration in SG of RGC. Several studies have demonstrated that the lymphatic pathway after distal gastrectomy differs from that in the original stomach [15, 22]. The important lymphatic flow and location of lymph node metastases in RGC are lymph drainage pathways along the left gastric artery and posterior gastric artery and along the anastomotic duodenum and jejunum. In patients with ERGC located near the anastomosis site, the main lymphatic pathway might be along the left gastric artery, along the anastomotic duodenum or jejunum and the left gastroepiploic artery. For ERGC located near the anastomosis site, and not indicated for endoscopic treatment, we employed SG with routine lymphadenectomy of stations 1, 3,7 , and $4 \mathrm{sb}$, and along the anastomotic duodenum or jejunum, in some cases, stations $8 \mathrm{a}$ and $11 \mathrm{p}$, similar to distal gastrectomy. In the TG group, there were two patients with lymph node metastasis. The lymph node stations were along the short gastric artery (station 4sa) and left paracardial lymph node (station 2); however, both tumors were located in the cardia. We employed SG for 13 patients with ERGC according to the preoperative diagnosis; 3 patients were found to have muscularis propria invasion. Since the left gastric artery remained in all patients in the SG group, we considered the main lymphatic pathway might be along the left gastric artery and along the anastomotic duodenum or jejunum. Therefore, there was no recurrence in the SG group.

Operating time, blood transfusion, and hospital stay were similar in the two groups; however, blood loss tended to be lower in the SG group. This might have been because blood loss was proportional to the area needed for synechotomy. Frequency of complications was similar in the two groups; however, the fact that there was no anastomotic leakage in the SG group was significant.

Gastrectomy usually causes postoperative complications, including dumping syndrome and reduced food intake, that lead to weight loss. There were no cases of no dumping syndrome in the SG group compared to three patients in the TG group. The decrease in food intake and weight loss might have occurred during the first operation in both groups; therefore, the change in body weight in the present study might not have been significant. With regard to nutritional status, we did not directly estimate food intake; however, we estimated laboratory data 1 year after remnant gastrectomy. Hemoglobin and total protein were significantly higher in the SG group. There was no need to inject vitamin B12 for the patients in the SG group because of the preserved stomach.

The present study demonstrated that SG for ERGC reduced the occurrence of dumping syndrome and improved anemia and total protein levels compared to TG. There was no recurrence of gastric cancer in the SG group. We adopted the SG for ERGC with the concept of distal gastrectomy after anterectomy. Although several authors have reported better outcomes after less invasive surgery for primary gastric cancer [17-21], there have been no reports on limited surgery for RGC. This study is believed to be the first on less invasive surgery for RGC. With careful selection of patients with ERGC, SG could be a safe and effective treatment compared to conventional TG. However, further studies of a larger number of patients with longer follow-up periods are necessary for a more definitive conclusion.

Acknowledgments No grants were received for this study.

Conflict of interest The authors declare that there were no conflicts of interest. 


\section{References}

1. Balfour DC. Factors influencing the life expectancy of patients operated on for gastric ulcer. Ann Surg. 1922;76:405-8.

2. Helsingen N, Hillestad L. Cancer development in the gastric stump after partial gastrectomy for ulcer. Ann Surg. 1956;143:170.

3. Domellof L, Eriksson S, Janunger KG. Late precancerous changes and carcinoma of the gastric stump after Bilroth I resection. Am J Surg. 1976;132:26.

4. Shafer LW, Larson DE, Melton LJ III, Higgins JA, IIstrup DM. The risk of gastric carcinoma after surgical treatment for benign ulcer disease: a population-based study in Olmsted Country, Minnesota. N Engl J Med. 1983;309:1210-13.

5. Ross AH, Smith MA, Anderson JR, Small WP. Late mortality after surgery for peptic ulcer. New Engl $J$ Med. 1982;307:519-22.

6. Lundegardh G, Adami HO, Helmick C, Zack M, Meirik O. Stomach cancer after partial gastrectomy for benign ulcer disease. N Engl J Med. 1988;319:195-200.

7. Fischer AB, Graem N, Jensen OM. Risk of gastric cancer after Billroth II resection for duodenal ulcer. $\mathrm{Br} \mathrm{J}$ Surg. 1983;70:552-54.

8. Sowa M, Onoda N, Nakanishi I, Maeda K, Yoshikawa K, Kato Y, et al. Early stage carcinoma of the gastric remnant in Japan. Anticancer Res. 1993;13:1835-8.

9. Greene FL. Management of gastric remnant carcinoma based on the results of a 15-year endoscopic screening program. Ann Surg. 1996;223:701-6.

10. Kaneko K, Kondo H, Saito D, Shirao K, Yamaguchi H, Yokota T, et al. Early gastric stump cancer following distal gastrectomy. Gut. 1998;43:342-4.

11. Takenaka R, Kawahara Y, Okada H, Tsuzuki T, Yagi S, Kato J, et al. Endoscopic submucosal dissection for cancers of the remnant stomach after distal gastrectomy. Gastrointest Endosc. 2008;67:359-63.
12. Japanese Gastric Cancer Association. Japanese classification of gastric carcinoma. Gastric Cancer. 1998;1:10-24.

13. Ohashi M, Katai H, Fukagawa T, Gotoda T, Sano T, Sasako M, et al. Cancer of the gastric stump following distal gastrectomy for cancer. Br J Surg. 2007;94:92-5.

14. Ahn HS, Kim JW, Yoo MW, Park Do J, Lee HJ, Lee KU, et al. Clinicopathological features and surgical outcomes of patients with remnant gastric cancer after a distal gastrectomy. Ann Surg Oncol. 2008;15:1632-9.

15. Hu X, Tian DY, Cao L, Yu Y. Progression and prognosis of gastric stump cancer. J Surg Oncol. 2009;100:472-6.

16. Tanigawa N, Nomura E, Lee SW, Kaminishi M, Sugiyama M, Aikou T, et al. Current state of gastric stump carcinoma in Japan: based on the results of a nationwide survey. World J Surg. 2010;34:1540-7.

17. Furukawa H, Hiratsuka M, Imaoka S, Ishikawa O, Kabuto $T$, Sasaki Y, et al. Phase II study of limited surgery for early gastric cancer: segmental gastric resection. Ann Surg Oncol. 1999;6:166-70.

18. Ohwada S, Nakamura S, Ogawa T, Izumi M, Tanahashi Y, Sato $\mathrm{Y}$, et al. Segmental gastrectomy for early cancer in the midstomach. Hepatogastroenterology. 1999;46:1229-33.

19. Seto Y, Yamaguchi H, Shimoyama S, Shimizu N, Aoki F, Kaminishi M. Results of local resection with regional lymphadenectomy for early gastric cancer. Am J Surg. 2001;182:498-501.

20. Shinohara T, Ohyama S, Muto T, Kato Y, Yanaga K, Yamaguchi T. Clinical outcome of high segmental gastrectomy for early gastric cancer in the upper third of the stomach. Br J Surg. 2006;93:975-80.

21. Ishikawa K, Arita T, Ninomiya S, Bandoh T, Shiraishi N, Kitano S. Outcome of segmental gastrectomy versus distal gastrectomy for early gastric cancer. World J Surg. 2007;31:2204-7.

22. Han SL, Hua YW, Wang CH, Ji SQ, Zhuang J. Metastatic pattern of lymph node and surgery for gastric stump cancer. J Surg Oncol. 2003;82:241-6. 